\title{
A importância do exercício físico em tempos de pandemia: uma revisão integrativa da literatura
}

\section{The importance of physical exercise in times of pandemic: an integrative literature review}

Recebimento dos originais: 01/05/2021

Aceitação para publicação: 31/06/2021

\section{Lorena Karen de Morais Moura Castro}

Centro Universitário Uninovafapi (UNINOVAFAPI), Teresina-Piauí.

Mariana de Sousa Meneses Carvalho

Centro Universitário Uninovafapi (UNINOVAFAPI), Teresina-Piauí.

\author{
Milena Viana Freire \\ Centro Universitário Uninovafapi (UNINOVAFAPI), Teresina-Piauí. \\ Miguel Henrique Pereira de Paiva \\ Universidade Federal do Piauí (UFPI), Teresina-Piauí. \\ Aureliano Machado de Oliveira \\ Centro Universitário Uninovafapi (UNINOVAFAPI), Teresina-Piauí.
}

\begin{abstract}
RESUMO
O estilo de vida ativo é de fundamental importância para manter a saúde, deve ser estimulada durante o período da pandemia devido aos muitos benefícios proporcionados pelo mesmo à saúde, principalmente ao sistema cardiovascular, metabólico e imunológico.
\end{abstract}

Palavras-chave: Exercício físico, COVID-19, Sedentarismo.

\begin{abstract}
The active lifestyle is of fundamental importance to maintain health, and should be encouraged during the pandemic period due to the many benefits it provides to health, especially to the cardiovascular, metabolic, and immune systems.
\end{abstract}

Keywords: Physical exercise, COVID-19, Sedentary lifestyle.

\section{INTRODUÇÃO}

A pandemia do COVID -19 colocou o mundo em estado de alerta, pois a carência de um tratamento específico e efetivo no agravamento da doença e a ausência de um processo de um agente imunizante estimulou a busca por um estado de bem-estar relacionado a saúde (ALBERTON, et al.,2020). Hábitos de higiene, uso de máscaras cirúrgicas e adoção de um distanciamento social colaboram de forma indireta para um colapso do sistema da saúde. (FERREIRA et al.,2020). 
O distanciamento social e a quarente decretada pelos gestores públicos ocasionou uma restrição da população as suas residências.Com jornada de trabalho e escola de forma remota, houve um aumento da ingestão alimentar e diminuição do gasto energético, desenvolvendo hábitos não saudáveis como o comportamento sedentário, onde todos esses fatores em consonância contribuem para o surgimento de comorbidades associadas ao maior risco cardiovascular, obesidade, aumento da pressão arterial, intolerância à glicose, bem como transtornos psicossociais como ansiedade e depressão, tornando o indivíduo susceptível ao agravamento da COVID-19.A prática do exercício físico deve ser estimulada durante o período da pandemia devidos as muitos benefícios proporcionados pelo mesmo à saúde, principalmente ao sistema cardiovascular, metabólico e imunológico (PELEGRINIDCP, et al. , 2020).

\section{OBJETIVO}

Identificar por meio de análise e revisão da literatura científica brasileira as evidências sobre a importância da prática do exercício físico como promotor da educação em saúde na pandemia do COVID19.

\section{MÉTODO}

A busca bibliográfica deu-se a partir de uma revisão integrativa utilizando como descritores: exercício físico, COVID-19, sedentarismo. Foram utilizados descritores em português, com artigos completos, entre os anos de 2020 e 2021.Foram utilizadas as bases de dados online Lilacs, Medline e Scielo.Como critérios de inclusão foram selecionados artigos completos, publicados em anais, periódicos que contemplasse a temática e como critérios de exclusão publicações artigos que apresentem alto níveis de plágio, em apreciação.

\section{REVISÃO BIBLIOGRÁFICA}

A busca bibliográfica reuniu 15 artigos, sendo selecionados apenas 2 para leitura na íntegra, levando em consideração os critérios de inclusão e exclusão.

É evidente o aumento da inatividade física e do comportamento sedentário devido a pandemia do Covid-19, assim como seus efeitos negativos à saúde decorrentes do isolamento social, pois é um fator de risco para as doenças crônicas não transmissíveis. Diante disso, a prática do exercício físico aeróbico, anaeróbico e atividades holísticas, como yoga e meditação devem ser estimuladas no ambiente domiciliar, utilizando recursos tecnológicos que favorecem o acompanhamento do treino de forma orientada e regular, pois promove, estimula e auxilia as pessoas durante a pandemia trazendo benefícios importantes à saúde física e mental (MATTOS MS,et al.,2020). 
A prática do exercício físico regular, adaptado a especificidade de cada indivíduo, melhora as condições de saúde aumentando a oxigenação cerebral, a síntese e a degradação de neurotransmissores, a liberação de serotonina, a viscosidade sanguínea, mantém o condicionamento físico, diminui a perda de massa óssea e muscular, aumenta a força, a coordenação e equilíbrio, reduz a incapacidade funcional, reduz a intensidade dos pensamentos negativos e das doenças físicas, promove a melhoria do bem-estar e do humor, além da melhora da pressão arterial, trazendo inúmeros benefícios relacionados aos distúrbios do sono, como insônia, melhorando aspectos cognitivos relacionados a memória e aprendizagem, ajuda na prevenção e controle de doenças crônico degenerativas. Dessa forma, a importância de manter um estilo de vida ativo mesmo em casa, traz benefícios fisiológicos e psicológicos, promovendo a qualidade de vida (BATISTA JI, e OLIVEIRA A,2015).

\section{DISCUSSÃO}

A pandemia do COVID-19 levou ao isolamento social onde teve como consequência a diminuição da prática de exercício físico e ao aumento do comportamento sedentário em adultos brasileiros. É muito importante enfatizar também a redução do tempo que ficamos sentados, deitados ou reclinados em frente a televisão ou computador e seus semelhantes, excetuando-se as horas de sono (PITANGA FJG, BECK CC $e$ PITANGA CPS,2020). O exercício físico de forma regular é indicado para manter a qualidade da saúde, sendo sugeridas a prática da caminhada, ciclismo, dança, yoga, musculação, treinamentos aeróbicos e de força, de modo a prevenir o desenvolvimento de doenças crônicas não transmissíveis, variando a intensidade e a duração do exercício (PRADO WL et al.,2020).

\section{CONSIDERAÇÕES FINAIS}

O exercício físico regular durante a pandemia do COVID-19 melhora a qualidade de vida, a saúde física e mental. É importante manter um estilo de vida ativo, tratando e prevenindo complicações das doenças crônico não transmissíveis. Sabe-se dos inúmeros benefícios que o treinamento físico praticado de forma regular traz, seja por aptidão física para força, equilíbrio, resistência no dia a dia, tendo como benefício o combate a inflamação crônica. Estimular a prática do exercício físico é necessário mesmo em situação de restrição distanciamento social atentando para os critérios de segurança e eficácia. 


\section{REFERÊNCIAS}

1. ALBERTON CL, et al. Exercício físico remoto e fadiga em sobreviventes do câncer de mama: uma intervenção em tempos da COVID-19. Rev Bras Ativ Fís Saúde.v.25, n.01, p.1-9;2020.

2. FERREIRA WL, et al. Ponto de vista dos profissionais de Educação Física sobre o uso da máscara facial durante o exercício físico na pandemia da COVID-19 Rev Bras Ativ Fís Saúde.v:25, n.1.p:1-9. 2020.

3. MATTOS,S.M.R et al.Recomendações de atividade física e exercício físico durante a pandemia Covid-19: revisão de escopo sobre publicações no Brasil.Rev.Bras.Ativ.Fis.Saude.v.25,n.76.2020.

4. MINAYO MCS, DESLANDES SF. Pesquisa social: teoria, método e criatividade.25 ed.rev. atual. Petrópolis:Vozes, 2007.

5. NOGUEIRA, IC et al. Efeitos do exercício físico no controle da hipertensão arterial em idosos: uma revisão sistemática. Rev.Bras.Geriatr.Gerontol., Rio de Janeiro. 15, n. 3, p. 587-601, Set. 2012.

6. PELEGRINI DCP, et al. Prática de atividade física em meio à pandemia da COVID-19: estudo de base populacional em cidade do sul do Brasil. Ciência \& Saúde Coletiva, n.25, v.11, p:4249-4258, 2020.

7. PITANGA FJG, BECK CC, PITANGA CPS. Atividade Física e Redução do Comportamento Sedentário durante a Pandemia do Coronavírus. Arq Bras Cardiol.n.114, v.6, p:1058-1060,2020.

8. PRADO, WL et al. Impacto da permanência em casa e do isolamento social, em função da COVID19, sobre o nível de atividade física e o comportamento sedentário em adultos brasileiros.Rev.Einstein.v.19, n.01.p.1-6.2021.6 